\title{
Virtual follow-up and care for patients with cardiac electronic implantable devices: protocol for a systematic review
}

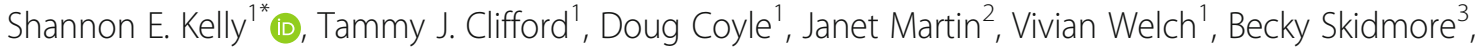

David Birnie ${ }^{4}$, Ratika Parkash ${ }^{5}$, Anthony S. L. Tang ${ }^{6}$ and George A. Wells ${ }^{1}$

\begin{abstract}
Background: Capacity to deliver outpatient care for patients with cardiac implantable electronic devices (CIEDs) may soon be outweighed by need. This systematic review aims to investigate the comparative effectiveness, safety, and cost for virtual or remote clinic interventions for patients with CIEDs and explores how outcomes may be influenced by patient or system factors in-depth.

Methods: We will perform a systematic literature search in MEDLINE, Embase, PsycINFO, CINAHL, Proquest Dissertations \& Theses, other EBM Reviews, and trial registry databases. Two authors will independently screen titles and abstracts for eligibility. We will include randomized and non-randomized controlled trials, quasi-randomized and experimental studies, cohort, and case-control studies. Study populations of interest are individuals with a CIED (pacemaker, ICD, CRT). Eligibility will be restricted to virtual or remote follow-up or care interventions compared to any other approach. The co-primary outcomes of interest are mortality and patient satisfaction. Secondary outcomes include clinical effectiveness (e.g., ICD shock, time-to-detection of medical event, hospitalizations), safety (e.g., serious or device-related adverse events), device efficacy (e.g., transmissions, malfunctions), costs, workflow (e.g., resources, process outcomes, time-saved), and patient reported (e.g., burden, quality of life). Data will be extracted by one author and checked by a second using a standardized template. We will use published frameworks to capture data relevant to intervention effects that may be influenced by intervention definition or complexity, context and setting, or in socially disadvantaged populations. Detailed descriptive results will be presented for all included studies and outcomes, and where feasible, synthesized using meta-analysis. Risk of bias will be assessed by two review authors independently using Cochrane Risk of Bias tools. Certainty of evidence will be assessed using the GRADE approach.
\end{abstract}

Discussion: Increases in number of CIEDs implanted, combined with an aging population and finite health resource allocations at the system-level may lead to increased reliance on virtual follow-up or care models in the future. These models must prioritize consistent, equitable, and timely care as a priority. Results from this systematic review will provide important insight into the potential contextual factors which moderate or mediate the effectiveness, safety, and cost of virtual follow-up or care models for patients.

(Continued on next page)

\footnotetext{
* Correspondence: skell102@uottawa.ca

${ }^{1}$ School of Epidemiology and Public Health, University of Ottawa, 101 - 600

Peter Morand Crescent, Ottawa, Ontario K1G 5Z3, Canada

Full list of author information is available at the end of the article
}

(c) The Author(s). 2020 Open Access This article is licensed under a Creative Commons Attribution 4.0 International License, which permits use, sharing, adaptation, distribution and reproduction in any medium or format, as long as you give appropriate credit to the original author(s) and the source, provide a link to the Creative Commons licence, and indicate if changes were made. The images or other third party material in this article are included in the article's Creative Commons licence, unless indicated otherwise in a credit line to the material. If material is not included in the article's Creative Commons licence and your intended use is not permitted by statutory regulation or exceeds the permitted use, you will need to obtain permission directly from the copyright holder. To view a copy of this licence, visit http://creativecommons.org/licenses/by/4.0/ The Creative Commons Public Domain Dedication waiver (http://creativecommons.org/publicdomain/zero/1.0/) applies to the data made available in this article, unless otherwise stated in a credit line to the data. 
(Continued from previous page)

Systematic review registration: PROSPERO registration number CRD42020145210

Keywords: Remote monitoring, Virtual follow-up, Virtual care, eHealth, Distance, Implantable device, Medical device, Models of care, Distance factors, Cardiac implantable electronic device, ICD, Pacemaker, CRT

\section{Background}

It is standard practice for patients with cardiovascular electronic devices (CIEDs) to visit specialty device outpatient clinics for follow-up; however, demands on these services may begin to outweigh available resources as populations age and more devices are being implanted $[1,2]$. Routine follow-up appointments can burden both patients and the outpatient clinics who manage followup care. Patients and their caregivers often travel long distances to attend appointments, and pacing clinics often struggle to manage the volume of in-clinic visits while maintaining optimal care for patients. Routine inclinic visits contribute to delays in access to appointment times for patients with greater clinical need or urgency. Over the last decade, advances in telecommunications and device technology have led to innovations in arrhythmia care [1]. Virtual outpatient visits (also known as remote interrogation or monitoring) are now used in many regions in a blended model combined with traditional in-clinic visits or as a means to follow patients more closely when they are subject to device recalls, alerts, or are close to requiring replacement of the device, leads, or battery [2]. In some jurisdictions, these virtual follow-up visits, alternated every 6 to 12 months with outpatient device clinic visits, for CIEDs are now considered standard of care $[2,3]$.

Virtual models of follow-up for pacemakers, implantable cardioverter defibrillators (ICDs), and cardiac resynchronization therapy (CRT) offer patients the convenience of staying in their own home while still obtaining quality care, a benefit that may especially be impactful to those living in rural or remote areas. Use of virtual surveillance and follow-up "visits" is often described using the term "remote monitoring." The value of virtual models of follow-up lies in the potential to monitor patients and their devices as effectively and safely as if they had attended an in-person appointment. Several randomized clinical studies have demonstrated that virtual follow-up is a broadly safe and beneficial alternative to in-person outpatient clinic visits in adult populations living with CIEDs, although there is less clinical evidence for pacemaker and CRT populations [4-8]. There is also interest in exploring the feasibility of eliminating routine in-clinic follow-up visits in favor of only virtual follow-up and surveillance. The RPM CIED randomized controlled trial currently underway in Canada is evaluating a virtual-only model for CRT, ICD, and pacemakers alongside a user-driver clinical application [9]. Likewise, researchers are studying if more complete virtual care (i.e., including some kind of virtual therapeutic intervention like device reprogramming or automatic calibration of devices using artificial intelligence) for patients can be implemented in patients with adequate battery longevity who are otherwise clinically stable [10-12]. This type of care intervention may also be referred to as "remote patient management" [12] and is not being used as part of standard care yet.

The umbrella phrase "remote monitoring" is used in both research and practice to describe the technology-enabled process where device or patient data and/or diagnostics are collected via passive remote device interrogations and automated transmission of active pre-specified alerts related to device functionality and clinical events are returned to a receptor device clinic. Although a well-recognized term in the clinical realm, it conceptually oversimplifies a clinical intervention which is quite complex [13]. Complex health interventions are usually defined as such because they are (a) multicomponent (i.e., comprised of multiple components that interact in potentially synergistic or discordant ways), (b) non-linear (they may not follow a simple causal pathway to bring about their effects), and (c) contextdependent (although not standardized, they may work best when tailored to local contexts) [14]. All three seem to apply directly to virtual follow-up or care for CIEDs, yet these nuances are often completely overlooked or undocumented in existing research. With virtual follow-up, complexity (1) comes from several interacting components (i.e., patient, device, transmission modality, software, user interfaces, clinic, education, and training), (2) involves groups or individuals at different organizational levels (i.e., patient, vendor, device clinic, institution), (3) has multiple and variable outcomes (e.g., related to device, patient, process, cost, equity), and (4) permits a certain degree of flexibility or tailoring [14].

Clinicians who support the use of virtual follow-up for patients with CIEDs have noted that outside of the research environment, there are issues with the delivery of timely, uniform, and efficient arrhythmia care in practice across health jurisdictions, and uptake is not optimal $[15,16]$. It is unclear if the health and system benefits identified in individual research studies (e.g., shorter time to detection and treatment of medical and devicerelated events, fewer clinic visits $[17,18])$ involving virtual follow-up for CIEDs are being realized in practice, 
and evidence syntheses have not found significant effects on cardiovascular or all-cause mortality, stroke, hospitalization when randomized studies are pooled. Results from these studies also demonstrate wide variation in both patient and device clinic setting along with intervention delivery characteristics related to how remote monitoring is offered to patients, the timing and frequency of follow-up, and data transmission and review which are not investigated further [18]. Recent surveys of both patients and device clinics in Canada have found that virtual follow-up falling under the "remote monitoring" catch-all is extremely heterogeneous in terms of who receives or is offered virtual follow-up; who, when, and how it is delivered; and how resulting data transmissions or alerts are handled [16].

Consequently, it seems overly simple to conclude that virtual follow-up for CIEDs is effective and safe based on an average effect across varying populations and delivery approaches. A lack of understanding about why or how complex virtual interventions work may lead to difficulties in quality improvement, implementation, adaptation, and scaling up of comparable interventions. We are currently unable to judge whether components within these approaches are effective and ineffective (or potentially unsafe), how approaches might be modified by contextual factors or setting, or if there are actual gains for patients in terms of increased accessibility to services and reach (e.g., ability to offer care to patients in rural or remote areas). Existing reviews fail to consider nonrandomized or observational study designs which may offer important contextual and organization of care details that offer potential insight and compliment randomized evidence. To scale, improve, and expand virtual follow-up or care offerings across often large and diverse populations and care settings, we need to better understand how context and different intervention components influence outcomes. The present systematic review protocol outlines our approach to comprehensively review the evidence for the comparative clinical effectiveness, safety, and cost-effectiveness of virtual follow-up or care models for patients with CIEDs while considering the complexity of the intervention and the system in which it interacts, and the impact of context and setting.

\section{Methods}

This protocol was written a priori and will be followed throughout the study process. Any deviations from this protocol will be disclosed in all related publications, and the PROSPERO record will be updated accordingly (CRD42020145210). All research questions will be addressed by a de novo systematic review of published clinical evidence. This systematic review protocol has been developed using developed methodological guidance for systematic reviews of complex health interventions [19-26] and considers guidance from the Preferred Reporting Items for Systematic Review and Meta-Analysis-Protocols (PRISMA-P) statement (Online Supplement 1) [27].

\section{Research questions}

The systematic review will address the primary research question: What are the comparative clinical effectiveness, safety, and cost of virtual follow-up or care models for patients with CIEDs?

Several subsets of the research question will be addressed to additionally capture the complexity of virtual follow-up or care and the context in which it is delivered:

1. What is virtual follow-up or care for CIEDs, and how is it defined?

2. How do the components of virtual follow-up/care interventions work alone or in combination to produce effects?

3. How does context affect the effects of the intervention or cost?

4. How do effects or cost change over time, and what explains change in the effects of the intervention over time?

5. What is the distribution of effects across socially disadvantaged populations [28]?

A qualitative evidence synthesis of patient and health care provider perspectives and care experiences, and implementation barriers is currently underway to supplement available evidence related to the research questions above (PROSPERO submission pending).

\section{Literature search strategy}

An experienced information specialist will design the literature search in consultation with the research team. The electronic search strategy will undergo peer-review by a second, independent information specialist using the Peer Review of Electronic Search Strategies (PRESS) Guideline Statement [29] prior to execution. The complete proposed search strategy is presented in Online Supplement 2.

Information will be identified by searching the following bibliographic databases: Using the OVID platform, Ovid MEDLINE ${ }^{\oplus}$, including Epub Ahead of Print and InProcess \& Other Non-Indexed Citations, Embase Classic + Embase, PsycINFO, and the following EBM Reviews databases: Cochrane Central Register of Controlled Trials, Cochrane Database of Systematic Reviews, Database of Abstracts of Reviews of Effects, Health Technology Assessment, and the NHS Economic Evaluation Database. We will also search CINAHL (EBSCO platform) and Proquest Dissertations \& Theses Global. 
An initial search strategy was designed and piloted between December 29, 2018, and January 6, 2019. The proposed strategy was run on June 26, 2019, to test for volume. The searches will be conducted in two parts. The main search will apply research design filters, and the second supplemental search will utilize an extensive qualitative filter. Qualitative and case studies will be the focus of the qualitative synthesis and will be reported separately. The core concepts in both parts will be comparable (i.e., remote monitoring and implantable cardiac devices). We will use a combination of controlled vocabulary (e.g., "Remote Consultation", "Defibrillators, Implantable", "Cardiac Electrophysiology") and key words (e.g., telemonitor, pacemaker, CIED) for the concepts in all searches. We will remove animal-only citations and news items where possible from the results. We plan on searching for relevant studies using ClinicalTrials.gov and the International Clinical Trials Registry Platform (ICTRP) Search Portal. Searches will be limited by date to records available after January 1, 2000 (to coincide with the first regulatory approvals of wireless, remote monitoring systems by Biotronik), and not limited in any other way (e.g., by language or publication status). Regular search updates will be performed on databases that do not provide alert services.

\section{Study eligibility}

The eligibility criteria relevant to addressing the research questions can be found in Table 1 .

The primary outcome will be all-cause mortality as this addresses both effectiveness and safety and because prevention of heart rhythm problems causing death is the ultimate treatment goal for any CIED. The coprimary outcome will be patient satisfaction (using any scale, instrument, or tool), as this outcome represents a patient-oriented care priority and may indicate willingness to continue with virtual follow-up or care. Studies will be included if they meet the selection criteria. No studies will be excluded based on the outcomes reported. In cases where studies report mixed populations (i.e., the study is comprised of individuals who do and do not meet the eligibility criteria), we will include studies that report results that apply to the population of interest separately. Where studies do not report results for the mixed population separately, we will include studies where the majority of the mixed population participants meet the eligibility criteria.

\section{Literature screening and selection}

Two independent reviewers will screen the titles and abstracts of all citations retrieved against the eligibility criteria (see Table 1). A citation will not be excluded during screening at the title and abstract stage unless both reviewers agree. All citations deemed by the reviewers to be potentially relevant (or unclear) at the title and abstract stage will be retrieved as full-text articles for a second level of screening by two independent review authors. Discrepancies will be resolved through discussion or consultation with a third reviewer if necessary. We will use standardized forms for article screening and selection set up within DistillerSR. The full literature screening and selection process will be documented and presented in a PRISMA flow diagram [30].

\section{Data extraction}

Information will be extracted from all included studies, including bibliographic information details pertinent to study characteristics (e.g., name of first author, publication year, publication title, country, funding sources), research methodology (e.g., study design, aim or objectives, inclusion and exclusion criteria, recruitment method, primary and secondary outcomes, subgroup analyses of interest), population (e.g., number of patients, age, sex/gender, race, type of device, cardiovascular disease severity, and baseline characteristics), and outcomes (e.g., definition, type of outcome, time of assessment, results for outcomes, and any subgroups). In addition, we will utilize three existing frameworks to guide data extraction as they will provide a template to guide data extraction and help to standardize the collection of information across included studies and amongst review authors, with consideration of appropriate levels of detail. This will help to better achieve the overall aim of finding evidence to answer the research questions, while also providing a transparent and reproducible methodology. The frameworks will be used to collect additional information from each included study relevant to the following: descriptions of the intervention and comparator models of follow-up or care (Template for Intervention Description and Replication [TIDieR] checklist [31]), influence of context and setting (Context and Implementation of Complex Interventions [CICI] framework [32]), or equity factors ([PROGRESS-Plus] framework [33, 34]) on the outcomes of interest (Table 2). Detailed data extraction tables are provided in Online Supplement 3.

The TIDieR framework is used to describe interventions in sufficient detail to allow for their replication [31]. Given the heterogeneity of approaches that can be used under the umbrella terms remote monitoring or management, this framework will assist with describing study intervention(s) in detail, and to consider the comparability of interventions described in the individual research studies included. The purpose of the CICI framework is to enable the systematic assessment and documentation of the context, setting, and implementation associated with complex interventions [32]. The purpose of the CICI framework is to enable the 
Table 1 Eligibility criteria for the research questions

\section{Population}

Adults or children (any gender) with a CIED (pacemaker, ICD, CRT). Device may be de novo, existing, or in a patient undergoing a pulse generator change that now has virtual follow-up or care capabilities.

Exclusions:

- Patients with implantable loop recorders.

\section{Intervention}

Virtual follow-up may also be referred to as remote monitoring. In this study we broadly include the collection of device or patient data and/or diagnostics via passive remote device interrogations and the automated transmission of active pre-specified alerts related to device functionality and clinical events. This involves a one-way transmission of data from the patient in their outpatient setting to a receptor device or specialty clinic. Here, the patient alternates virtual follow-up from home with inperson device clinic visits (6 month intervals for ICDs/CRT, 12 month intervals for pacemakers). This can be utilized for any CIED (pacemakers, ICDs, or CRTs) which have the capability. Virtual follow-up is most often suited for patients with stable device function and adequate battery longevity after at least one in-person post-surgical follow-up visit.

Virtual care may also be referred to as remote patient management and involves therapeutic intervention on the patient's implanted CIED from a distance using available technology (e.g., remotely re-programming device thresholds, automated recalibration of device settings using machine-learning algorithms). This involves twoway interaction and is informed by virtual follow-up through transmission of data from patient in their outpatient (or non-device clinic) setting to a receptor device clinic and then related care or action involving some kind of therapeutic adjustment from a physician (or qualified individual) at the receptor device clinic back to the patient's device. This eliminates the need for travel to a specialty device clinic, with exceptions for changes in medical status, battery replacement, or re-implant. Follow-up remains the same from home but patient may be required to travel from home to a local provider for virtual care intervention. This approach may also be accompanied by additional interventions aimed at patient self-efficacy and empowerment (e.g., providing a patient with their own data). Any virtual therapeutic intervention for CIED patients is considered in-scope for this review.

Exclusions:

- Biometric, non-device-related patient data (e.g., blood pressure, weight) follow-up via telecardiology.

\section{Comparators}

Any (standard care, in-clinic care, transtelephonic monitoring, a different virtual follow-up, or care model ${ }^{\mathrm{a}}$ )

\section{Outcomes $^{\mathrm{b}}$}

Primary outcomes: mortality (all), patient satisfaction with care

\section{Secondary outcomes:}

Clinical effectiveness: Cardiovascular mortality, stroke, ICD shocks (total, appropriate, inappropriate), arrhythmias (pacemaker patients only), time to detection of medical events (time to detection of ventricular arrhythmias, time to detection of atrial fibrillation, time to initiation of anti-coagulation in patients with new device-detected atrial fibrillation, time to detection of device infection, time to detection of device malfunction [including lead malfunction], time from detected medical event to action/decision, time to detection of suboptimal biventricular pacing (defined as $<90 \%$ in CRT patients only)), worsening NYHA functional class, response to CRT, total clinic visits (total, scheduled, unscheduled), emergency department visits, hospitalizations (all, device-related, for heart failure, or of cardiovascular cause), length of hospital stay, patient compliance, patient adherence.

Patient-reported or important: Activities of daily living, burden, self-efficacy, quality of life, empowerment

Device efficacy: system set-up (attempts, success, failure), transmissions (total, unsuccessful, successful), malfunctions with system, alerts, or data Safety: adverse events attributable to intervention, serious adverse events

Costs: any cost or resource use data identified will be of interest (related to the patient, clinic, or system). These must be economic elements, expressed as quantifiable outcomes or change in outcomes.

Workflow/operational: staff/physician resources or time, time per "appointment," process efficiencies, use of time for education or technical training, proportion of clinic patients using virtual follow-up or care, telephone calls made (by type if reported).

Other: Study withdrawals, group crossovers, composite endpoints involving any of the above outcomes. Equity in terms of geographic/socio-economic access or reach, impact to PROGRESS+ factors.

\section{Study design}

Randomized controlled trials, non-randomized controlled trials, quasi-randomized studies ${ }^{c}$, cohort studies (prospective, retrospective, historical) ${ }^{d}$, case-control studies, quasi-experimental studies.

Studies may be from peer-reviewed publications, trial registry records, conference abstracts, letters, presentations, or thesis/dissertation documents. Protocols and trial registry records that meet eligibility criteria will be included, investigated for publication bias, and will be documented or summarized as indicators of "in progress" research.

Exclusions: Case reports, case series, review articles, cross-sectional studies, surveys, qualitative or interview/focus group studies, editorials, letters, and commentaries.

\section{Context/setting}

Any setting or context.

All outcomes will be explored for meaningful differences measured by contextual factors, or those related to the setting of the device clinic or patient. Differences in PROGRESS-PLUS factors will be of interest, as well as any specified stratified reporting of findings, or evidence of moderating or mediating effects of context or setting.

\section{Time frame}

January 1, 2000, to present.

\section{Language}

No limitations.

CIED cardiovascular implantable electronic device, ICD implantable cardioverter defibrillator, CRT cardiac resynchronization therapy

a"A different virtual follow-up or care model" means any virtual follow-up or care model compared with any other virtual follow-up or care model (i.e., virtual follow-up or care models compared with each other)

${ }^{\mathrm{b}}$ All outcomes are considered to be important based on the scoping exercises that informed this review and involved consultation with more than one clinical expert in cardiology and electrophysiology

${ }^{\mathrm{C}} \mathrm{A}$ quasi-experimental design is an empirical interventional study used to associate outcomes of an intervention on a group of sampled participants without random assignment. For example, pretest-posttest, and interrupted time-series designs

${ }^{\mathrm{d}}$ Cohort studies are defined as studies in which a defined group of participants (the cohort) are sampled on the basis of exposure (interventions received) and in which subsequent outcomes and the association with different interventions received are assessed in a follow-up period of time. This is differentiated from case series studies, in which observations are made on a series of participants, usually all receiving the same intervention and who are followed over a defined time to examine subsequent outcomes, without a control group 
Table 2 Checklist and frameworks for data extraction

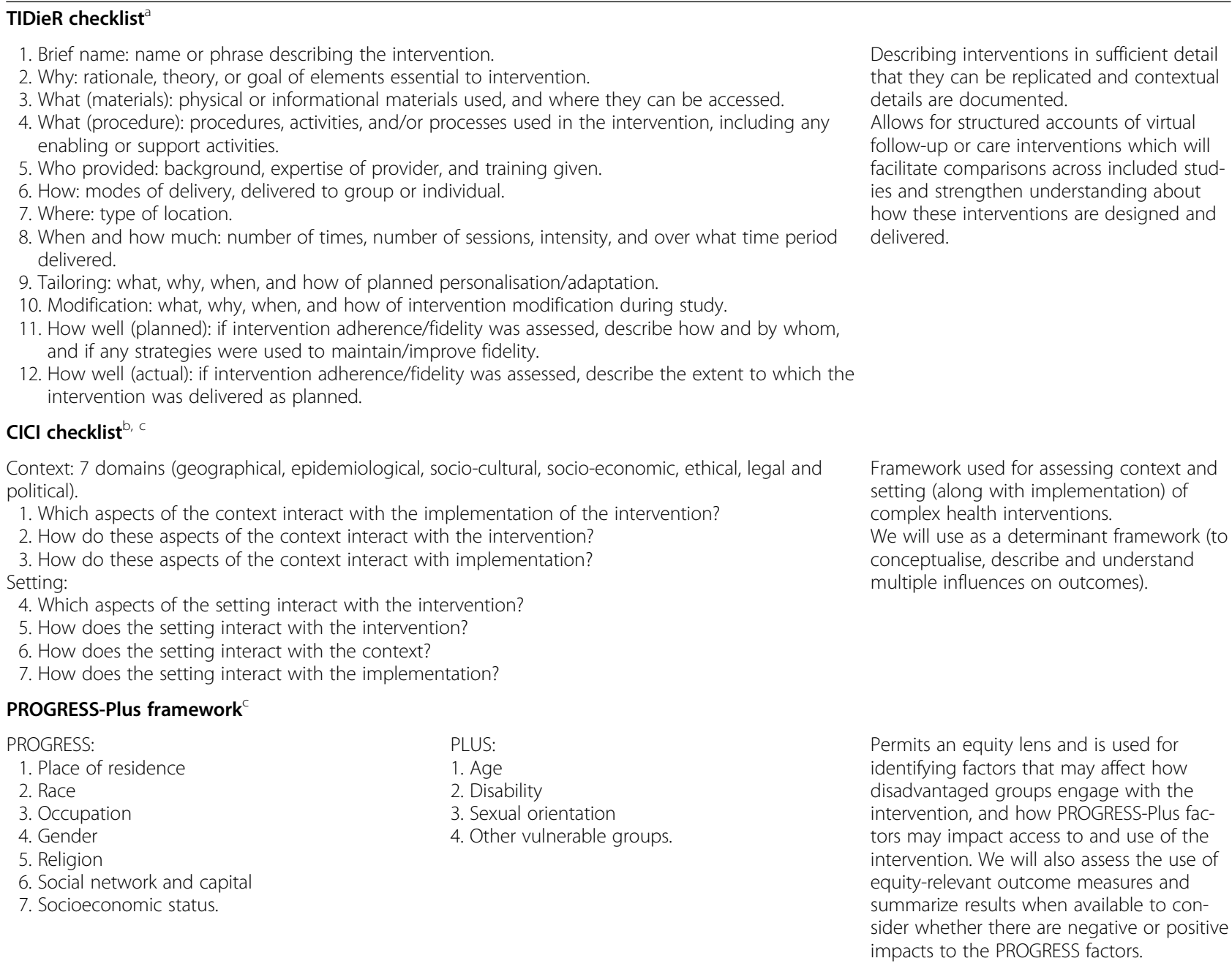

${ }^{a}$ Adapted from Hoffmann et al. 2014: Better reporting of interventions: template for intervention description and replication (TIDieR) checklist and guide ${ }^{b}$ Adapted from Pfadenhauer LM, Gerhardus A, Mozygemba K, Lysdahl KB, Booth A, Hofmann B, et al. Making sense of complexity in context and implementation: the context and implementation of complex interventions (CICl) framework. Implement Sci. 2017;12(1):21

${ }^{\mathrm{C}} \mathrm{CICl}$ items related to implementation activities and intervention description will not be extracted. Intervention descriptions will be extracted using TIDieR checklist items. Implementation experience will be documented in a separate systematic review and qualitative synthesis

${ }^{\mathrm{d}}$ Adapted from O'Neill J, Tabish H, Welch V, Petticrew M, Pottie K, Clarke M, et al. Applying an equity lens to interventions: using PROGRESS ensures consideration of socially stratifying factors to illuminate inequities in health. Journal of clinical epidemiology. 2014;67(1):56-64

systematic assessment and documentation of the context, setting, and implementation associated with complex interventions [32]. This overarching framework outlines potential interacting dimensions of context (including setting) and implementation using eight domains of context (i.e., setting, geographical, epidemiological, socio-cultural, socio-economic, ethical, legal, and political). There are also four implementation domains (i.e., provider, organisation and structure, funding, and policy). Implementation domains will not be a focus for data-extraction in the current review. The PROGRESS framework is used by the Cochrane and Campbell equity group to consider social determinants of heath [34]. PROGRESS stands for place of residence, race/ethnicity/ culture/language, occupation, gender/sex, religion, education, socioeconomic status, and social capital. The "Plus" represents added characteristics also associated with potential social disadvantage such as personal characteristics (e.g., age and disability), features of relationships, and time-dependent circumstances (e.g., refugee status) (Table 2).

All data extraction will be completed by one reviewer and checked for completeness and accuracy in its entirety by a second reviewer. Where data are only available from figures (no explicit numerical data are reported) in an included study, we will contact corresponding authors using information listed in the publication to obtain the missing data. Extraction of data for all included studies will be facilitated using forms in Microsoft Excel customized for this systematic review and standardized 
in advance. Where there are multiple reports for a single study, we will extract data from all reports into one form and document the related citations.

\section{Quality assessment and risk of bias for individual studies}

A risk of bias assessment will be conducted by two independent reviewers, and all disagreements will be resolved through discussion or consultation with a third reviewer as needed. Narrative summaries of all studies included will highlight the strengths and limitations, and tables or figures will be used to present and graphically summarize results. We will assess risk of bias using tools suggested by the Cochrane Collaboration [35], including the Risk of Bias tool [36], and the Risk of Bias in Nonrandomized Studies -of Interventions (ROBINS-I) tool [37]. The CLARITY risk of bias tool will be used for case-control studies [38].

\section{Certainty of evidence}

The Grading of Recommendations Assessment, Development, and Evaluation (GRADE) framework will be used to rate the certainty of the evidence of intervention effects $[39,40]$. Evidence from all studies will inform the assessment by a single review author. A second independent review author will verify results. A third independent reviewer will adjudicate any differences. Additional GRADE guidance currently under development in an ongoing research project will be used to more specifically contextualize the assessment for considerations relevant to complex health interventions [22]. This approach facilitates incorporation of evidence from the TIDieR, CICI, and PROGRESS-Plus frameworks into the GRADE assessment, and outlines additional considerations relating to context, setting, and other factors that assessors can use in rating the certainty of evidence. Results will be presented in Summary of Findings tables and accompanied with a narrative summary by outcome.

\section{Analysis \\ Descriptive syntheses}

Study characteristics and findings will be summarized descriptively and presented in tabular format. Results from all included studies will be summarized and synthesized narratively.

\section{Meta-analyses}

Where feasible and appropriate, we will pool the results of included studies using meta-analyses. Feasibility will be based on the availability of sufficient data for each outcome while appropriateness will be assessed through reviewer evaluation of the available data for clinical, methodological, and statistical heterogeneity. Evaluations will be informed through consultation with a biostatistician (statistical and methodological homogeneity) and clinical practitioners (clinical and methodological homogeneity) in cardiology and electrophysiology. Decisions will be made as to the important patient or design factors that may be expected to modify or mediate the outcomes of interest. Results from interventions aimed at monitoring or surveillance of patients and their devices (i.e., virtual follow-up or remote monitoring) will be analyzed separately from interventions where virtual follow-up is combined with virtual therapeutic care (i.e., virtual care or remote patient management).

The results from different study designs will not be pooled. Rather, results will be pooled in separate metaanalyses according to study design. No meta-analyses will be conducted for any study without a control group. Random effects meta-analyses will be performed using the Cochrane Review Manager software (RevMan version 5.3 or the version available at the time). The unit of analysis will be the participant. All dichotomous outcomes (e.g., mortality, stroke) will be pooled using relative risks (randomized, cohort studies, quasiexperimental, or quasi-randomized) or odd ratios (case control studies), and 95\% confidence intervals (CIs) will be calculated. Pooling of continuous outcomes will be done using mean differences and corresponding 95\% CIs or standardized mean differences where, for example, outcome scales differ. Where adjusted results are reported in effect measures, the analyses will use adjusted results. Differences between unadjusted and adjusted results will be investigated where data are sufficient and discussed. When results are not presented with appropriate measures of variance, the variances will be imputed where possible. If medians or quartiles are reported, estimates of means and associated standard deviations will be calculated [41].

Individual and pooled estimates from all meta-analyses will be presented using tables, and forest plots will be created. Additional visualization approaches may also be used (e.g., harvest or albatross plots [26]). Statistical heterogeneity will be measured and evaluated using forest plots and calculations of Cochran's chi-square test and the $I^{2}$ statistic during meta-analyses, and guidance from the Cochrane Handbook will be used to interpret heterogeneity [35].

\section{Subgroup and meta-regression analyses}

Subgroup analyses are a straightforward and flexible approach appropriate for evaluating the impact of context, setting, participants, intervention characteristics, and other factor (e.g., equity) influences on outcomes [26]. Where feasible and appropriate, we will use subgroup analyses to explore all of these factors, identifying as many subgroups of interest as possible a priori, and 
using exploratory analyses to investigate the impact of subgroups identified during the review. The a priori subgroups of interest to be analysed in meta-analyses are as follows:

- Type of device (pacemaker, ICD, CRT);

- Vendor (Medtronic, Abbott, Boston Scientific, Biotronik, LivaNova, or others if listed);

- Severity and type of underlying cardiovascular disease (e.g., disease, New York Heart Association (NYHA) Functional Classification I through IV);

- Remoteness to device clinic (urban, rural, remote);

- Type of residence (e.g., home/personal residence, assisted living residence, long-term care home);

- Length of time using virtual follow-up or care $(<1$ year, $\geq 1$ to $<2$ years, $\geq 2$ to $<5$ years, 5 years or more);

- Country of delivery;

- Indication for use of virtual follow-up or care (general patient management, safety alert or recall, battery or device replacement imminent).

The impact of important variations in intervention delivery or design identified using the TIDieR checklist will be investigated through subgroup analyses where possible. A priori subgroups of interest related to variation in delivery or design include frequency of transmission (daily, weekly, monthly), follow-up and circle of care (where, by whom), education and training components (by minutes spent educating patient), clinic response to transmissions or alerts, and any key differences identified through TIDieR items. Intervention descriptions will also be used to assess the potential for interventiongenerated inequities [28].

All context and setting variables identified using the CICI framework will be subject to subgroup analyses (Table 2). Meta-analyses of the patient, device clinic, and systems levels' factors will consider impacts to important outcomes at the micro, meso, and macro scales of the context and setting domains [32]. The CICI framework may also capture important aspects of the PROGRESSPlus factors. All PROGRESS-Plus items will be investigated through subgroup analyses. The credibility of subgroup analyses will be assessed using criteria by Sun et al. using information from each study relevant to the design, analysis, and context (Online Supplement 4) [42]. Where there is a paucity of data to inform analyses based on these factors, they will be considered in the interpretation of the review.

Where the number of studies is sufficient, metaregression analyses may be used to examine the effects of multiple study features relevant to context, setting, participants, or intervention characteristics, or to explore the mediating effects of intermediate outcomes.

\section{Sensitivity analyses}

Sensitivity analyses may be considered to evaluate the robustness of pooled estimates using methodological and statistical aspects, including the impact of study design (e.g., randomized controlled trials versus cohort studies versus case-control studies), study quality, date of publication, composition of the study population (i.e., $100 \%$ meet eligibility criteria versus "mixed" populations), or varying definitions used for study outcomes.

\section{Publication bias}

Visual tools (e.g., Funnel plots for RCTs) and statistical tests (e.g., Egger's regression test, Begg's rank correlation test) will be used to assess publication bias where there are 10 or more included randomized studies [35].

\section{Reporting}

This systematic review will aim to meet all criteria outlined in A Measurement Tool to Assess Systematic Reviews revised tool (AMSTAR 2) [43]. Relevant reporting guidelines will be considered when preparing the written report of systematic review methods and findings (PRISMA [30], PRISMA harms [44], PRISMA equity [45], and the Meta-analysis Of Observational Studies in Epidemiology [MOOSE] checklist [46]). Consideration will also be given to reporting guidance relevant specifically to the TIDieR [31], CICI [32], and PROGRESS-Plus [34] frameworks used in this review.

\section{Amendments}

Any amendments to this protocol will be documented, reflected in edits to the PROSPERO registration, and presented with the findings of the review.

\section{Dissemination}

The results from this systematic review will be published in a peer-reviewed journal and disseminated through various media, including, but not limited to conferences, seminars, congresses, or symposia.

\section{Discussion}

Increases in number of devices implanted combined with an aging population and finite health resource allocations at the system-level may lead to increased reliance on virtual follow-up or care models in the future, while prioritizing consistent, equitable, and timely care as a priority. This systematic review will provide important insight into the potential contextual factors which moderate or mediate the effectiveness, safety, and cost of virtual follow-up or care models for patients with implanted cardiac electronic devices. It will summarize hypothesized variation in studied intervention design and delivery to improve our understanding of this model of follow-up and care, while considering an equity lens 
to investigate variables that may impact access to and use of healthcare interventions.

Potential threats to this review are the large number of non-RCT studies that may be included, although we believe that their inclusion is necessary to capture important contextual, setting, and other details that may not have been described or of interest in published trials. There is also a possibility that there will be a paucity of quantitative data to answer some of the research questions of interest. Using the TIDieR, CICI and PROGRESS-PLUS frameworks will facilitate descriptive synthesis in this case, and gaps in knowledge to answer any of the current research questions will provide a foundation for additional quantitative and qualitative primary research.

Considerations for context, setting, and equity are necessary given the complex nature of virtual follow-up and care, and the reciprocal complexity of the health care system in which it is used. Results will be used to identify factors in individuals, intervention design, delivery, or potential implementation contexts or settings leading to success in terms of outcomes for the patient. The findings will inform future work that will aim to improve access, uptake, use, and spread of virtual follow-up and care models for CIED patients that will be applicable to inform improvements in post-implant care in both high or low and middle income countries. Ideally, new practice guidelines for management of this population will be developed that adequately consider all evidence relevant to the delivery of context and equity-sensitive patient-centred arrhythmia management strategies for virtual follow-up and care.

Provenance and peer review Not commissioned; externally peer reviewed.

\section{Supplementary information}

Supplementary information accompanies this paper at https://doi.org/10. 1186/s13643-020-01406-6.

Additional file 1. PRISMA-P checklist.

Additional file 2. Proposed search strategy

Additional file 3. Proposed data extraction form.

Additional file 4. Subgroup analyses criteria.

\section{Abbreviations}

AMSTAR 2: Assessing the Methodological Quality of Systematic Reviews (version 2); CICl: Context and Implementation of Complex Interventions; Cl: Confidence interval; CIED: Cardiac implantable electronic device; CRT: Cardiac resynchronization therapy; GRADE: Grading of Recommendations, Assessment, Development and Evaluations; ICD: Implantable cardioverter defibrillator; MOOSE: Meta-analyses of Observational Studies in Epidemiology; PRISMA: Preferred Reporting Items for Systematic Reviews and Meta-Analyses; PROGRESS-Plus: Place of residence, race/ethnicity, occupation, gender, religion/culture, education, socioeconomic status, social capital/networks, plus other important factors which impact on health equity; RCT: Randomized controlled trial; TIDieR: Template for Intervention Description and Replication

\section{Authors' contributions}

The manuscript was drafted by SK. WW, JM, DC, TC, RP, DB, AT, and GW substantially contributed to the development of conception and design, and BS to the section on searching and the development of the search strategy. All authors reviewed the manuscript critically for important intellectual content. All authors read and approved the final manuscript.

\section{Funding}

Shannon Kelly is funded partially through a graduate student bursary from the Cardiac Arrhythmia Network of Canada (CANet). The funding body had no role in the design of the study and collection, analysis, and interpretation of data or in writing the manuscript.

Availability of data and materials

Not applicable

Ethics approval and consent to participate

Not applicable

Consent for publication

Not applicable

\section{Competing interests}

Ratika Parkash reports research grants from Medtronic Canada and Abbott Canada. All remaining authors declare that they have no competing interests.

\section{Author details}

${ }^{1}$ School of Epidemiology and Public Health, University of Ottawa, 101 - 600 Peter Morand Crescent, Ottawa, Ontario K1G 5Z3, Canada. ${ }^{2}$ Department of Epidemiology and Biostatistics, Western University, London, Ontario, Canada. ${ }^{3}$ Independent Information Specialist, Ottawa, Ontario, Canada. ${ }^{4}$ University of Ottawa Heart Institute, Ottawa, Ontario, Canada. ${ }^{5}$ Division of Cardiology, Dalhousie University, Halifax, Nova Scotia, Canada. ${ }^{6}$ Schulich School of Medicine and Dentistry, Western University, London, Ontario, Canada.

Received: 25 October 2019 Accepted: 7 June 2020

Published online: 27 June 2020

\section{References}

1. Cheung CC, Deyell MW. Remote monitoring of cardiac implantable electronic devices. Can J Cardiol. 2018;34(7):941-4.

2. Yee R, Verma A, Beardsall M, Fraser J, Philippon F, Exner DV. Canadian Cardiovascular Society/Canadian Heart Rhythm Society joint position statement on the use of remote monitoring for cardiovascular implantable electronic device follow-up. Can J Cardiol. 2013;29(6):644-51.

3. Slotwiner D, Varma N, Akar JG, Annas G, Beardsall M, Fogel Rl, et al. HRS expert consensus statement on remote interrogation and monitoring for cardiovascular implantable electronic devices. Heart rhythm. 2015;12(7):e69e100.

4. Varma N, Michalski J, Epstein AE, Schweikert R. Automatic remote monitoring of implantable cardioverter-defibrillator lead and generator performance: the Lumos-T Safely RedUceS RouTine Office Device Follow-Up (TRUST) trial. CircArrhythmElectrophysiol. 2010;3(5):428-36.

5. Crossley $\mathrm{GH}$, Boyle A, Vitense H, Chang Y, Mead RH, Connect I. The CONNECT (Clinical Evaluation of Remote Notification to Reduce Time to Clinical Decision) trial: the value of wireless remote monitoring with automatic clinician alerts. J Am CollCardiol. 2011:57(10):1181-9.

6. Hindricks G, Taborsky M, Glikson M, Heinrich U, Schumacher B, Katz A, et al. Implant-based multiparameter telemonitoring of patients with heart failure (IN-TIME): a randomised controlled trial. Lancet. 2014;384(9943):583-90.

7. Guedon-Moreau L, Lacroix D, Sadoul N, Clementy J, Kouakam C, Hermida JS, et al. A randomized study of remote follow-up of implantable cardioverter defibrillators: safety and efficacy report of the ECOST trial. Eur Heart J. 2013; 34(8):605-14.

8. Mabo P, Victor F, Bazin P, Ahres S, Babuty D, Da CA, et al. A randomized trial of long-term remote monitoring of pacemaker recipients (the COMPAS trial). Eur Heart J. 2012;33(9):1105-11. 
9. García-Fernández FJ, Osca Asensi J, Romero R, Fernández Lozano I, Larrazabal JM, Martínez Ferrer J, et al. Safety and efficiency of a common and simplified protocol for pacemaker and defibrillator surveillance based on remote monitoring only: a long-term randomized trial (RM-ALONE). Eur Heart J. 2019;40(23):1837-46.

10. Clinical Trials Registration (clinicaltrials.gov) for Remote patient management for patients with CIEDs (RPM-CIED TACHY) 2019. Available from: https:// clinicaltrials.gov/ct2/show/NCT03405740?term=remote+patient+ management\&rank=1. Accessed 12 Mar 2020.

11. Clinical Trials Registration (clinicaltrials.gov) for Remote patient management for patients with CIEDs (RPM-CIED BRADY) 2019. Available from: https:// clinicaltrials.gov/ct2/show/NCT03636230?term=remote+patient+ management\&rank=3. Accessed 12 Mar 2020.

12. Clinical Trials Registration (clinicaltrials.gov) for Remote Patient Management for Cardiac Implantable Electronic Devices (RPM-CIED) - A pilot study 2019 Available from: https://clinicaltrials.gov/ct2/show/NCT02585817. Accessed 12 Mar 2020

13. Norris SL, Rehfuess EA, Smith H, Tunçalp Ö, Grimshaw JM, Ford NP, et al. Complex health interventions in complex systems: improving the process and methods for evidence-informed health decisions. BMJ Global Health. 2019;4(Suppl 1):e000963.

14. Craig P, Dieppe P, Macintyre S, Michie S, Nazareth I, Petticrew M. Developing and evaluating complex interventions: the new Medical Research Council guidance. BMJ. 2008;337:a1655.

15. Piccini JP, Mittal S, Snell J, Prillinger JB, Dalal N, Varma N. Impact of remote monitoring on clinical events and associated health care utilization: a nationwide assessment. Heart rhythm. 2016;13(12):2279-86.

16. Campbell D, editor Cardiac implantable electronic device remote monitoring follow up: informing future guidelines and policy from the patients' perspective. Canadian Heart Rhythm Society Annual Meeting (Complex Device Management session); 2019 September 13, 2019; Montreal, Quebec.

17. Parthiban N, Esterman A, Mahajan R, Twomey DJ, Pathak RK, Lau DH, et al. Remote monitoring of implantable cardioverter-defibrillators: a systematic review and meta-analysis of clinical outcomes. J Am CollCardiol. 2015;65(24):2591-600.

18. Remote monitoring of implantable cardioverter-defibrillators, cardiac resynchronization therapy and permanent pacemakers: a health technology assessment. Ont Health Technol Assess Ser. 2018;18(7):1-199.

19. Booth A, Moore G, Flemming K, Garside R, Rollins N, Tunçalp Ö, et al. Taking account of context in systematic reviews and guidelines considering a complexity perspective. BMJ Global Health. 2019;4(Suppl 1):e000840.

20. Booth A, Noyes J, Flemming K, Moore G, Tunçalp Ö, Shakibazadeh E. Formulating questions to explore complex interventions within qualitative evidence synthesis. BMJ Global Health. 2019;4(Suppl 1):e001107.

21. Flemming K, Booth A, Garside R, Tunçalp Ö, Noyes J. Qualitative evidence synthesis for complex interventions and guideline development: clarification of the purpose, designs and relevant methods. BMJ Global Health. 2019; 4(Suppl 1):e000882.

22. Montgomery P, Movsisyan A, Grant SP, Macdonald G, Rehfuess EA. Considerations of complexity in rating certainty of evidence in systematic reviews: a primer on using the GRADE approach in global health. BMJ Global Health. 2019;4(Suppl 1):e000848.

23. Noyes J, Booth A, Moore G, Flemming K, Tunçalp Ö, Shakibazadeh E. Synthesising quantitative and qualitative evidence to inform guidelines on complex interventions: clarifying the purposes, designs and outlining some methods. BMJ Global Health. 2019:4(Suppl 1):e000893.

24. Petticrew M, Knai C, Thomas J, Rehfuess EA, Noyes J, Gerhardus A, et al. Implications of a complexity perspective for systematic reviews and guideline development in health decision making. BMJ Global Health. 2019; 4(Suppl 1):e000899.

25. Rehfuess EA, Stratil JM, Scheel IB, Portela A, Norris SL, Baltussen R. The WHO-INTEGRATE evidence to decision framework version 1.0: integrating WHO norms and values and a complexity perspective. BMJ Global Health. 2019;4(Suppl 1):e000844.

26. Higgins JPT, López-López JA, Becker BJ, Davies SR, Dawson S, Grimshaw JM, et al. Synthesising quantitative evidence in systematic reviews of complex health interventions. BMJ Global Health. 2019;4(Suppl 1):e000858.

27. Moher D, Shamseer L, Clarke M, Ghersi D, Liberati A, Petticrew M, et al. Preferred reporting items for systematic review and meta-analysis protocols (PRISMA-P) 2015 statement. Syst Rev. 2015;4:1.
28. Welch VA, Petkovic J, Jull J, Hartling L, Klassen T, Kristjansson E, Pardo Pardo J, Petticrew M, Stott DJ, Thomson D, Ueffing E, Williams K, Young C, Tugwel P. Chapter 16: Equity and specific populations. In: Higgins JPT, Thomas J, Chandler J, Cumpston M, Li T, Page MJ, Welch VA (editors). Cochrane Handbook for Systematic Reviews of Interventions version 6.0 (updated July 2019). Cochrane, 2019. Available from www.training.cochrane.org/ handbook. Accessed 17 June 2020

29. McGowan J, Sampson M, Salzwedel DM, Cogo E, Foerster V, Lefebvre C. PRESS Peer Review of Electronic Search Strategies: 2015 Guideline Statement. J Clin Epidemiol. 2016;75:40-6.

30. Moher D, Liberati A, Tetzlaff J, Altman DG. Preferred reporting items for systematic reviews and meta-analyses: the PRISMA statement. J Clin Epidemiol. 2009;62(10):1006-12.

31. Hoffmann TC, Glasziou PP, Boutron I, Milne R, Perera R, Moher D, et al. Better reporting of interventions: template for intervention description and replication (TIDieR) checklist and guide. BMJ. 2014;348:g1687.

32. Pfadenhauer LM, Gerhardus A, Mozygemba K, Lysdahl KB, Booth A, Hofmann B, et al. Making sense of complexity in context and implementation: the Context and Implementation of Complex Interventions (CICl) framework. Implement Sci. 2017;12(1):21.

33. Whitehead $M$. The concepts and principles of equity and health. Int J Health Serv. 1992;22(3):429-45.

34. O'Neill J, Tabish H, Welch V, Petticrew M, Pottie K, Clarke M, et al. Applying an equity lens to interventions: using PROGRESS ensures consideration of socially stratifying factors to illuminate inequities in health. J Clin Epidemiol. 2014;67(1):56-64.

35. Cochrane Handbook for Systematic Reviews of Interventions, version 6.0. Higgins JPT TJ, Chandler J, Cumpston M, Li T, Page MJ, Welch VA, editor: Cochrane (John Wiley \& Sons); 2019.

36. Higgins JPT, Savović J, Page MJ, Elbers RG, Sterne JAC. Chapter 8: Assessing risk of bias in a randomized trial. In: Higgins JPT, Thomas J, Chandler J, Cumpston M, Li T, Page MJ, Welch VA (editors). Cochrane Handbook for Systematic Reviews of Interventions version 6.0 (updated July 2019). Cochrane, 2019. Available from www.training.cochrane.org/handbook. Accessed 17 June 2020.

37. Sterne JA, Hernán MA, Reeves BC, Savović J, Berkman ND, Viswanathan M, et al. ROBINS-l: a tool for assessing risk of bias in non-randomised studies of interventions. Bmj. 2016;355:14919.

38. Partners E. Tool to assess risk of bias in cohort studies/case control studies/ randomized controlled trials from the CLARITY Group at McMaster University. 2019 [Methods Resources]. Available from: https://www.evidencepartners.com/ resources/methodological-resources/. Accessed 12 Mar 2020.

39. Guyatt G, Oxman AD, Akl EA, Kunz R, Vist G, Brozek J, et al. GRADE guidelines: 1. Introduction-GRADE evidence profiles and summary of findings tables. J Clin Epidemiol. 2011;64(4):383-94.

40. Schunemann HJ, Cuello C, Akl EA, Mustafa RA, Meerpohl JJ, Thayer K, et al. GRADE guidelines: 18. How ROBINS-I and other tools to assess risk of bias in nonrandomized studies should be used to rate the certainty of a body of evidence. J Clin Epidemiol. 2019;111:105-14

41. Wan X, Wang W, Liu J, Tong T. Estimating the sample mean and standard deviation from the sample size, median, range and/or interquartile range. BMC Med Res Methodol. 2014;14:135

42. Sun X, Briel M, Walter SD, Guyatt GH. Is a subgroup effect believable? Updating criteria to evaluate the credibility of subgroup analyses. BMJ. 2010;340:C117.

43. Shea BJ, Reeves BC, Wells G, Thuku M, Hamel C, Moran J, et al. AMSTAR 2: a critical appraisal tool for systematic reviews that include randomised or nonrandomised studies of healthcare interventions, or both. BMJ. 2017;358:;4008

44. Zorzela L, Loke YK, loannidis JP, Golder S, Santaguida P, Altman DG, et al. PRISMA harms checklist: improving harms reporting in systematic reviews. BMJ. 2016;352:1157.

45. Welch V, Petticrew M, Tugwell P, Moher D, O'Neill J, Waters E, et al. PRISMAEquity 2012 extension: reporting guidelines for systematic reviews with a focus on health equity. PLoS Med. 2012;9(10):e1001333.

46. Stroup DF, Berlin JA, Morton SC, Olkin I, Williamson GD, Rennie D, et al. Meta-analysis of observational studies in epidemiology: a proposal for reporting. Meta-analysis Of Observational Studies in Epidemiology (MOOSE) group. JAMA. 2000;283(15):2008-12.

\section{Publisher's Note}

Springer Nature remains neutral with regard to jurisdictional claims in published maps and institutional affiliations. 